\title{
Shape Projectors for Landmark-Based Spline Curves
}

\author{
Daniel Schmitter and Michael Unser
}

\begin{abstract}
We present a generic method to construct orthogonal projectors for two-dimensional landmark-based parametric spline curves. We construct vector spaces that define a geometric transformation (e.g., affine, similarity, and scaling) that is applied to a reference curve. These vector spaces contain all parametric curves up to the chosen transformation. We define the vector spaces implicitly through an orthogonal projection operator and present a theorem that characterizes the projector for landmark-based spline curves, which are popular for the user-interactive analysis of biomedical images. Finally, we show how shape priors are constructed with the spline projector and provide an example of application for the segmentation of microscopy images in biology.
\end{abstract}

Index Terms-Landmarks, projector, shape prior, splines.

\section{INTRODUCTION}

W E CONSIDER the problem of aligning a query curve $r$ defined in the continuous domain as $\boldsymbol{r}(t)=\left(r_{x}(t), r_{y}(t)\right)$ with $t \in \mathbb{R}$, onto a reference shape $r^{\mathrm{ref}}$. Thereby, we allow $\boldsymbol{r}^{\mathrm{ref}}$ to deform as $\boldsymbol{r}^{\text {ref }} \mapsto \mathcal{T}_{\boldsymbol{\theta}}\left\{\boldsymbol{r}^{\mathrm{ref}}\right\}$ according to a subclass of geometric transformations $\mathcal{T}_{\boldsymbol{\theta}}$ that belong to the affine family (see Table I) parameterized by $\boldsymbol{\theta}$. To that end, we consider the generic parametric alignment problem

$$
\min _{\boldsymbol{\theta}}\left\|\mathcal{T}_{\boldsymbol{\theta}}\left\{\boldsymbol{r}^{\mathrm{ref}}(t)\right\}-\boldsymbol{r}(t)\right\|_{L_{2}}^{2} .
$$

Problem (1) arises in image segmentation settings [1]-[4] when prior knowledge is integrated [5]-[7] such that the solution should be close to a given shape [8], [9]. For specific transformations where a particular structure is enforced on $\mathcal{T}_{\boldsymbol{\theta}}$ several algorithms have been proposed [10]-[13]. In [14], we have presented a solution for the specific case $r \mapsto \Theta r+\rho$ with $\Theta \in \mathbb{R}^{2 \times 2}$ and $\rho \in \mathbb{R}^{2}$, which is not generalizable for the arbitrary case. Here, we derive a closed-form solution of (1) that is applicable to any particular type of transformation of the affine family. Our solution includes the classical case described in procrustean analysis [15], [16] but we are able to retrieve the sought-after similarity transformation in one step. Moreover, we propose an exact spline-based implementation of our continuous-domain framework. We provide a generic solution by defining a finite-dimensional vector space that contains all possible shapes obtained by a given transformation of $r^{\mathrm{ref}}$ defined by $\mathcal{T}_{\boldsymbol{\theta}}$. We characterize a vector space as a subspace that contains all shapes that are related to a reference shape by a specific transformation. The subspace itself is implicitly characterized by its orthogonal projector.

Manuscript received June 21, 2017; revised August 7, 2017; accepted August 20,2017. Date of publication August 24, 2017; date of current version September 8 , 2017. This work was supported by the Swiss National Science Foundation under Grant 200020-162343/1. The associate editor coordinating the review of this manuscript and approving it for publication was Dr. Antonio Paiva. (Corresponding author: Daniel Schmitter.)

The authors are with the Biomedical Imaging Group, École polytechnique fédérale de Lausanne, Lausanne 1015, Switzerland (e-mail: daniel. schmitter@epfl.ch; michael.unser@epfl.ch).

Color versions of one or more of the figures in this letter are available online at http://ieeexplore.ieee.org.

Digital Object Identifier 10.1109/LSP.2017.2743692
This allows us to compute the "best match" among curves defined by a subspace w.r.t. an arbitrary shape. Intuitively speaking, projecting a query shape onto the subspace defined by the reference amounts to "choosing" the reference up to a specific transformation that is closest to the input shape. We propose solutions for continuously defined parametric spline curves. They are popular for interactive applications, where a user manually enforces landmark correspondence to increase robustness. Other applications, where invariance to parameterization is required have been studied in [17]-[19].

\section{Vector Spaces Defining Affine Shape Spaces}

\section{A. Hilbert Space $\mathcal{H}$ Containing All Parametric Curves}

We denote by $\mathcal{H}: L_{2}\left([0,1], \mathbb{R}^{2}\right)$ the Hilbert space that contains all parametric curves and is associated with the standard $L_{2}$-inner product $\left\langle\boldsymbol{r}_{i}, \boldsymbol{r}_{j}\right\rangle_{L_{2}}:=\int_{0}^{1} \boldsymbol{r}_{i}^{\mathrm{T}}(t) \boldsymbol{r}_{j}(t) \mathrm{d} t$. The corresponding norm is defined as $\|\boldsymbol{r}\|_{L_{2}}:=\sqrt{\langle\boldsymbol{r}, \boldsymbol{r}\rangle_{L_{2}}}$. Without loss of generality, we restrict the parameter domain to $t \in[0,1]$.

\section{B. Vector Spaces as Subspaces of $\mathcal{H}$}

We define a subspace that contains all specific affine transformations of the reference curve $r^{\text {ref }}$. It can be defined as the finitedimensional vector space $\mathcal{S}^{\text {ref }}$ of dimension $I$, whose basis consists of elements $\left\{\boldsymbol{e}_{i}^{\mathrm{ref}}\right\}_{i=1, \ldots, I}$, which themselves are curves that depend on $\boldsymbol{r}^{\text {ref }}$. Every element (i.e., curve) living in $\mathcal{S}^{\text {ref }}$ can be expressed as a linear combination of the basis elements. Thus, $S^{\mathrm{ref}}=\left\{\sum_{i=1}^{I} u_{i} \boldsymbol{e}_{i}^{\mathrm{ref}}(\cdot): u_{i} \in \mathbb{R}\right\}$ is a subspace of the Hilbert space $\mathcal{H}$.

1) Example: A linear mapping of a 2-D curve can be expressed as $\boldsymbol{\Theta} r$, where $\boldsymbol{\Theta}=\left(\begin{array}{cc}\theta_{1} & \theta_{2} \\ \theta_{3} & \theta_{4}\end{array}\right)$ is a $(2 \times 2)$ matrix with elements $\theta_{i} \in \mathbb{R}$. By explicitly evaluating the matrix-vector product, we obtain

$$
\begin{aligned}
\boldsymbol{\Theta} \boldsymbol{r}(t)= & \theta_{1}\left(\begin{array}{c}
r_{x}(t) \\
0
\end{array}\right)+\theta_{2}\left(\begin{array}{c}
r_{y}(t) \\
0
\end{array}\right) \\
& +\theta_{3}\left(\begin{array}{c}
0 \\
r_{x}(t)
\end{array}\right)+\theta_{4}\left(\begin{array}{c}
0 \\
r_{y}(t)
\end{array}\right) .
\end{aligned}
$$

Therefore, the subspace associated to the two-dimensional (2-D) reference curve $\boldsymbol{r}^{\mathrm{ref}}$ is a 4 -D vector space (i.e., $I=4$ ) whose basis is given by

$$
\left\{\boldsymbol{e}_{i}^{\mathrm{ref}}\right\}_{i \in[1, \ldots, 4]}=\left\{\left(\begin{array}{c}
r_{x}^{\mathrm{ref}} \\
0
\end{array}\right),\left(\begin{array}{c}
r_{y}^{\mathrm{ref}} \\
0
\end{array}\right),\left(\begin{array}{c}
0 \\
r_{x}^{\mathrm{ref}}
\end{array}\right),\left(\begin{array}{c}
0 \\
r_{y}^{\mathrm{ref}}
\end{array}\right)\right\}
$$

where we have omitted the continuous parameter $t$ to simplify the notation. Note that the choice of the basis is not unique. However, different bases w.r.t. to a given transformation describe the same space.

\section{Construction of Vector Spaces}

In Table I we present examples of bases $\left\{\boldsymbol{e}_{i}\right\}_{i=1, \ldots, I}$ that can be used to construct a subspace $\mathcal{S}^{\text {ref }}$ for specific 2-D transformations. 
TABLE I

EXAMPLE BASES FOR 2-D VeCTOR SPACES

\begin{tabular}{|c|c|c|}
\hline Transformation & Degrees of Freedom & Basis $\left\{\boldsymbol{e}_{i}^{\text {ref }}\right\}$ w.r.t. $\boldsymbol{r}^{\text {ref }}$ \\
\hline Uniform scaling & 1 & \\
\hline Nonuniform scaling & 2 & \\
\hline Translation & 2 & \\
\hline Uniform scaling + Rotation & 2 & \\
\hline Similarity (uniform scaling + rotation + translation) & 4 & \\
\hline Linear mapping & 4 & \\
\hline Affine & 6 & \\
\hline Shear + Uniform scaling & 2 & \\
\hline Shear + Uniform scaling + Translation & 4 & \\
\hline Shear + Uniform scaling & 2 & \\
\hline Shear + Uniform scaling + Translation & 4 & \\
\hline Reflection + Uniform scaling + Rotation & 2 & \\
\hline Reflection + Similarity & 4 & \\
\hline
\end{tabular}

Taking a reference curve $\boldsymbol{r}^{\mathrm{ref}}=\left(r_{x}^{\mathrm{ref}}, r_{y}^{\mathrm{ref}}\right)$ and choosing a transformation given in Table I, the corresponding vector space is given by the indicated basis.

\section{LANDMARK-BASED PARAMETRIC SPline CURVES}

We consider curves of the form

$$
\boldsymbol{r}(t)=\left(\begin{array}{l}
r_{x}(t) \\
r_{y}(t)
\end{array}\right)=\sum_{n=0}^{N-1} \boldsymbol{c}[n] \varphi_{n}(t)
$$

where $\varphi$ is a compactly supported spline-based generator function and $N \in \mathbb{N}_{+}$represents the number of control points of the curve. The spline coefficients are given by $\{\boldsymbol{c}[k]=$ $\left.\left(c_{x}[k], c_{y}[k]\right)\right\}_{k=0, \ldots, N-1}$. The following algorithm can be easily generalized for the case where the summation limits are different than in (2). The sum in (2) is finite, which implies that the number of nonzero coefficients $c[k]$ is finite too. From (2), we see that the curve is constructed through integer shifts (represented by $n$ ) of the generator $\varphi$. Furthermore, for the model (2) to be affine invariant, the generator $\varphi$ needs to be capable of reproducing constants as specified by Proposition 1 . in (2)

Proposition 1: For $\Theta \in \mathbb{R}^{2 \times 2}, \boldsymbol{\rho} \in \mathbb{R}^{2}$, and a curve $\boldsymbol{r}$ as defined

$$
1 \in \operatorname{span}\left\{\varphi_{n}\right\} \Rightarrow \boldsymbol{\Theta} \mathbf{r}(t)+\boldsymbol{\rho} \in \operatorname{span}\left\{\varphi_{n}\right\}
$$

holds.
The proof follows immediately by noticing that, for any constant $\rho \in \mathbb{R}$, the relation $1 \in \operatorname{span}\left\{\varphi_{n}\right\} \Rightarrow \rho \in \operatorname{span}\left\{\varphi_{n}\right\}$ holds. Hence, any translation vector $\rho \in \mathbb{R}^{2}$ can be represented by (2) while the linearity of the model ensures that $\Theta \boldsymbol{r}(t) \in \operatorname{span}\left\{\varphi_{n}\right\}$.

\section{A. Construction of Vector Spaces Using Spline Curves}

To guarantee a unique representation of a spline curve (2) by its control points as well as a stable implementation, $\varphi$ needs to generate a Riesz basis [20] as, for instance, polynomial B-splines do. In that case, we can express the vector spaces defined in Section II-C as a function of the control points of the reference curves. For a curve $\boldsymbol{r}(t)=\left(r_{x}(t), r_{y}(t)\right)$ we define $\mathbf{c}=\left(\mathbf{c}_{x}, \mathbf{c}_{y}\right)$, which is a vector of length $2 N$, where

$$
\mathbf{c}_{x}=\left(c_{x}[0], \ldots, c_{x}[N-1]\right)
$$

contains the $N$ coefficients that describe $x(t)$ and the vector $\mathbf{c}_{y}$ that defines $y(t)$ is constructed in a similar way. Observe that we use regular bold font to describe the $\mathbf{c}$ in (5) as opposed to italic bold font to describe the different object $c$ in (2).

1) Example-Affine Transformation Combined With Translation: If we include a translation $\rho \in \mathbb{R}^{2}$ in the transformation described in Section II-B1, then we obtain $\Theta r+\rho$, where $\Theta$ is defined in Section II-B1 and $\boldsymbol{\rho}=\left(\theta_{5}, \theta_{6}\right)$. The transformation has six degrees of freedom and it is easy to see that a basis for the vector 
space of the reference curve $r^{\text {ref }}$ is given by

$$
\begin{aligned}
\left\{\boldsymbol{e}_{i}^{\mathrm{ref}}\right\}_{i \in[1, \ldots, 6]}= & \left\{\left(\begin{array}{c}
r_{x}^{\mathrm{ref}} \\
0
\end{array}\right),\left(\begin{array}{c}
r_{y}^{\mathrm{ref}} \\
0
\end{array}\right),\left(\begin{array}{c}
0 \\
r_{x}^{\mathrm{ref}}
\end{array}\right),\left(\begin{array}{c}
0 \\
r_{y}^{\mathrm{ref}}
\end{array}\right),\right. \\
& \left.\left(\begin{array}{l}
1 \\
0
\end{array}\right),\left(\begin{array}{l}
0 \\
1
\end{array}\right)\right\}
\end{aligned}
$$

which corresponds to

$$
\begin{aligned}
\left\{\mathbf{c}_{\boldsymbol{e}_{i}^{\mathrm{ref}}}\right\}_{i \in[1, \ldots, 6]}= & \left\{\left(\begin{array}{c}
\mathbf{c}_{x} \\
\mathbf{0}
\end{array}\right),\left(\begin{array}{c}
\mathbf{c}_{y} \\
\mathbf{0}
\end{array}\right),\left(\begin{array}{c}
\mathbf{0} \\
\mathbf{c}_{x}
\end{array}\right),\left(\begin{array}{c}
\mathbf{0} \\
\mathbf{c}_{y}
\end{array}\right),\right. \\
& \left.\left(\begin{array}{c}
\mathbf{1} \\
\mathbf{0}
\end{array}\right),\left(\begin{array}{c}
\mathbf{0} \\
\mathbf{1}
\end{array}\right)\right\}
\end{aligned}
$$

where 0 and 1 correspond to the vectors of size $N$ (which is the size of $\mathbf{c}_{x}$ or $\mathbf{c}_{y}$ ) and whose elements are all 0 or 1 , respectively. The spline projector is then computed by the application of Theorem 1 .

\section{IV. $L_{2}$-InNER Product of SPLine-BASEd CURVES}

Next, we derive a simple and efficient expression to compute the $L_{2}$-inner product $\left\langle\boldsymbol{r}_{1}, \boldsymbol{r}_{2}\right\rangle_{L_{2}}$ between spline-based curves. We first compute it for the 1-D case and then generalize it to higher dimensions.

\section{A. Inner Product}

First, we consider spline-based 1-D functions of the form $x(t)=$ $\sum_{n=0}^{N-1} c_{x}[n] \varphi_{n}(t)$. The $L_{2}$-inner product is then expressed as follows:

$$
\begin{aligned}
\left\langle x_{1}, x_{2}\right\rangle_{L_{2}} & =\int_{0}^{1} x_{1}(t) x_{2}(t) \mathrm{d} t \\
& =\sum_{m=0}^{N-1} \sum_{n=0}^{N-1} c_{1 x}[m] c_{2 x}[n]\left\langle\varphi_{m}, \varphi_{n}\right\rangle_{L_{2}} .
\end{aligned}
$$

We collect all the coefficients of the function $x$ in the vector of length $N$ as specified by (3). We then define $[\boldsymbol{\Phi}]_{m, n}=\left\langle\varphi_{m}, \varphi_{n}\right\rangle_{L_{2}}$. Now, (4) is expressed as $\left\langle x_{1}, x_{2}\right\rangle_{L_{2}}=\mathbf{c}_{1 x}^{\mathrm{T}} \boldsymbol{\Phi} \mathbf{c}_{2 x}$, where $\boldsymbol{\Phi}$ is the $(N \times N)$ autocorrelation matrix of $\varphi$. For an implementation (4) can be crucial. The entries of the matrix $\Phi$ can be precomputed and stored in a look-up table for fast online evaluation. The acceleration is considerable. It is due to the fact that the computation of the integral associated with the inner product (4) boils down to a matrix-vector multiplication.

Similarly, we simplify the 2-D inner product. By using the notations introduced in Section III-A the corresponding inner product is now expressed as follows:

$$
\left\langle\boldsymbol{r}_{1}, \boldsymbol{r}_{2}\right\rangle_{L_{2}}=\mathbf{c}_{1}^{\mathrm{T}} \boldsymbol{\Psi} \mathbf{c}_{2}=\left\langle\mathbf{c}_{1}, \mathbf{c}_{2}\right\rangle_{\boldsymbol{\Psi}}
$$

where

$$
\Psi=\left[\begin{array}{ll}
\Phi & 0 \\
0 & \Phi
\end{array}\right]
$$

and $\mathbf{0}$ is a zero matrix with the same dimensions as $\boldsymbol{\Phi}$.

\section{Main Result: Generalized Spline Projectors}

Using the simplified expression (5) to compute inner products of spline curves, we can now specify the projection operator. A fundamental aspect of our construction is not only that the curve $r$ that is being projected is a spline curve, but also that the basis $\left\{\boldsymbol{e}_{i}^{\mathrm{ref}}\right\}$ of the subspace $\mathcal{S}^{\text {ref }}$ consists of spline curves of the form given by (2). Hence, each of these curves $r \in \mathcal{H}$ is uniquely determined by its corresponding vector of control points $\mathbf{c} \in \mathbb{R}^{2 N}$.

In this letter, we provide the expression for 2-D curves, noting that the extension to 3-D is straightforward. We first define the matrix $\mathbf{C}^{\text {ref }}=\left[\mathbf{c}_{\boldsymbol{e}_{1}^{\text {ref }}} \cdots \mathbf{c}_{\boldsymbol{e}_{I}^{\text {ref }}}\right]$. It has the dimension $(2 N \times I)$ and contains the control points of the curves $\left\{\boldsymbol{e}_{i}^{\mathrm{ref}}\right\}_{i=1, \ldots, I}$ that define a basis of $\mathcal{S}^{\text {ref }}$. To simplify the notation, we collect all basis functions in the vector $\varphi(t):=\left(\varphi_{0}(t), \ldots, \varphi_{N-1}(t)\right)$. The corresponding (orthogonal) spline projector $\mathcal{P}^{\text {ref }}: \mathcal{H} \rightarrow \mathcal{S}^{\text {ref }}$ that minimizes the distance between the curve $r \in \mathcal{H}$ and the $I$-dimensional vector space $\mathcal{S}^{\text {ref }}$ is specified by Theorem 1 .

Theorem 1: Let $\boldsymbol{r}(t)=\boldsymbol{\varphi}(t)^{\mathrm{T}} \boldsymbol{c}$. Then

$$
\mathcal{P}^{\mathrm{ref}} \boldsymbol{r}(t)=\left(\begin{array}{cc}
\boldsymbol{\varphi}(t) & \mathbf{0} \\
\mathbf{0} & \boldsymbol{\varphi}(t)
\end{array}\right)^{\top} \mathbf{P}^{\mathrm{r} e f} \mathbf{c}
$$

where $\mathbf{P}^{\text {ref }} \in\left(\mathbb{R}^{2 N} \times \mathbb{R}^{2 N}\right): \mathbb{R}^{2 N} \rightarrow \mathbb{R}^{2 N}$ is the $(2 N \times 2 N)$ projection matrix defined as follows:

$$
\mathbf{P}^{\text {ref }}=\mathbf{C}^{\text {ref }}\left(\mathbf{C}^{\text {ref }}{ }^{T} \boldsymbol{\Psi} \mathbf{C}^{\text {ref }}\right)^{-1} \mathbf{C}^{\text {ref }}{ }^{T} \boldsymbol{\Psi} .
$$

Proof: If $\mathcal{P}$ is an orthogonal projector w.r.t. $\operatorname{span}\left\{\boldsymbol{e}_{i}\right\}_{i=1, \ldots, I}$, then a curve $r$ can always be decomposed as follows:

$$
\boldsymbol{r}(t)=\mathcal{P} \boldsymbol{r}(t)+\underbrace{(\mathcal{I}-\mathcal{P}) \boldsymbol{r}(t)}_{\text {error }}
$$

where $\mathcal{I}$ is the identity operator and the error between the curve and the projective plane is orthogonal to the projective plane, so that error $\perp \operatorname{span}\left\{\boldsymbol{e}_{i}\right\}$. By expressing $\mathcal{P} \boldsymbol{r}=\sum_{i}\left\langle\tilde{\boldsymbol{e}}_{i}, \boldsymbol{r}\right\rangle_{L_{2}} \boldsymbol{e}_{i}(t)=$ $\sum_{i} u_{i} \boldsymbol{e}_{i}(t)$ in (7) and taking the inner product on both sides w.r.t. $e_{k}$, we obtain the normal equation:

$$
\left\langle\boldsymbol{r}, \boldsymbol{e}_{k}\right\rangle_{L_{2}}=\sum_{i} u_{i}\left\langle\boldsymbol{e}_{i}, \boldsymbol{e}_{k}\right\rangle_{L_{2}}+\underbrace{\left.\langle\mathcal{I}-\mathcal{P}) \boldsymbol{r}(t), \boldsymbol{e}_{k}\right\rangle_{L_{2}}}_{0} .
$$

Evaluating (8) for all elements of the basis $\left\{\boldsymbol{e}_{k}\right\}$ and writing all the equations in matrix form, we obtain

$$
\left(\begin{array}{c}
\left\langle\boldsymbol{r}, \boldsymbol{e}_{1}\right\rangle_{L_{2}} \\
\vdots \\
\left\langle\boldsymbol{r}, \boldsymbol{e}_{I}\right\rangle_{L_{2}}
\end{array}\right)=\mathbf{G u} \Leftrightarrow \mathbf{u}=\mathbf{G}^{-1}\left(\begin{array}{c}
\left\langle\boldsymbol{r}, \boldsymbol{e}_{1}\right\rangle_{L_{2}} \\
\vdots \\
\left\langle\boldsymbol{r}, \boldsymbol{e}_{I}\right\rangle_{L_{2}}
\end{array}\right)
$$

Using the notation $\mathbf{E}=\left[e_{1} \cdots e_{I}\right], \mathbf{G}=\mathbf{C}^{\mathrm{T}} \boldsymbol{\Psi} \mathbf{C}$ being the Gram matrix with respect to the basis $\left\{\boldsymbol{e}_{i}\right\}$ and $\boldsymbol{g}=$ $\left(\left\langle\boldsymbol{e}_{1}, \boldsymbol{r}\right\rangle_{L_{2}}, \ldots,\left\langle\boldsymbol{e}_{I}, \boldsymbol{r}\right\rangle_{L_{2}}\right)$, the orthogonal projection of $\boldsymbol{r}$ is expressed as follows:

$$
\mathcal{P} \boldsymbol{r}(t)=\mathbf{E G}^{-1} \boldsymbol{g}=\sum_{i=1}^{I} \boldsymbol{e}_{i}(t) \underbrace{\left\langle\mathbf{G}_{i, 1}^{-1} \boldsymbol{e}_{1}+\cdots+\mathbf{G}_{i, I}^{-1} \boldsymbol{e}_{I}, \boldsymbol{r}\right.}_{\left\langle\tilde{\boldsymbol{e}}_{i}, \boldsymbol{r}\right\rangle_{L_{2}}}\rangle_{L_{2}}
$$

where $\left\{\tilde{\boldsymbol{e}}_{i}\right\}$ forms the dual basis of $\left\{\boldsymbol{e}_{i}\right\}$ and is defined as $\tilde{\mathbf{E}}=$ $\mathbf{E}\left(\mathbf{G}^{-1}\right)^{\mathrm{T}}=\left[\tilde{\boldsymbol{e}_{1}} \cdots \tilde{\boldsymbol{e}_{I}}\right]$. Because $\varphi$ generates a Riesz basis, each coordinate function of a spline curve $r$ given by (2) is uniquely specified by its control points $\{\mathbf{c}[k]\}_{k \in \mathbb{Z}}$. This implies that there is a one-to-one relation between the coordinate functions of the curve and its spline coefficients. Hence, the matrix $\mathbf{E}$ in (9) that defines the basis $\left\{\boldsymbol{e}_{i}\right\}$ for the subspace $\mathcal{S}$ (not to be confused with the Riesz basis generated by $\varphi$ ) is fully specified by $\mathbf{C}=\left[\mathbf{c}_{1} \cdots \mathbf{c}_{I}\right]$, which is the matrix that contains all the control points of the basis $\left\{\boldsymbol{e}_{i}\right\}$. Using (5), we rewrite (9) as follows:

$$
\mathcal{P} \boldsymbol{r}(t)=\mathbf{E G}^{-1} \boldsymbol{g}=\mathbf{E G}^{-1}\left(\left\langle\boldsymbol{e}_{1}, \boldsymbol{r}\right\rangle_{L_{2}}, \ldots,\left\langle\boldsymbol{e}_{I}, \boldsymbol{r}\right\rangle_{L_{2}}\right)
$$


which is equivalent to

$$
\mathbf{C G}^{-1}\left(\mathbf{c}_{1}^{\mathrm{T}} \boldsymbol{\Psi}, \ldots, \mathbf{c}_{I}^{\mathrm{T}} \boldsymbol{\Psi}\right) \mathbf{c}=\mathbf{C G}^{-1} \mathbf{C}^{\mathrm{T}} \boldsymbol{\Psi} \mathbf{c}=\mathbf{P} \mathbf{c} .
$$

Hence, $\mathbf{P}=\mathbf{C}\left(\mathbf{C}^{\mathrm{T}} \boldsymbol{\Psi} \mathbf{C}\right)^{-1} \mathbf{C}^{\mathbf{T}} \boldsymbol{\Psi}$. It is easily verified that $\mathbf{P}$ is a projector, characterized by the idempotent relation $\mathbf{P}^{2}=\mathbf{P}$.

Theorem 1 provides a direct method to compute the control points of the projected curve. Note that the projection of the vector $\mathbf{c}$ of control points is itself not orthogonal. However, it corresponds to the orthogonal projection of $\boldsymbol{r}$ in the $L_{2}$-sense. Therefore, we have $\left(\mathbf{P}^{\text {ref }}\right)^{2}=\mathbf{P}^{\text {ref }}$ and $\mathbf{P}^{\text {ref }}{ }^{T} \neq \mathbf{P}^{\text {ref }}$. Hence, Theorem 1 shows that $\mathbf{P}^{\text {ref }}$ is an oblique projector from $\mathbb{R}^{2 N}$ onto the $I$-dimensional invariant subspace of $\mathbb{R}^{2 N}$ defined by the basis $\left\{\mathbf{c}_{\boldsymbol{e}_{1}^{\mathrm{ref}}}\right\}_{i=1, \ldots, I}$. This means that $\mathcal{P}^{\text {ref }}: \mathcal{H} \rightarrow \mathcal{S}^{\text {ref }}$, which is the orthogonal projector in the $L_{2}$-sense, is efficiently implemented via the oblique projector $\mathbf{P}^{\text {ref }} \in\left(\mathbb{R}^{2 N} \times \mathbb{R}^{2 N}\right): \mathbb{R}^{2 N} \rightarrow \mathbb{R}^{2 N}$

\section{A. Example}

We show how the projectors that correspond to the vector spaces listed in Table I are implemented using splines, in accordance with Theorem 1. The simplest case is the scaling projector, which can be expressed by solving $\min _{a}\left\|a \boldsymbol{r}^{\text {ref }}-\boldsymbol{r}\right\|_{L_{2}}^{2}$ such that $\mathcal{P}^{\text {ref }} \boldsymbol{r}(t)=a \boldsymbol{r}^{\text {ref }}$, where $r^{\text {ref }}$ is the reference curve that defines the vector space. Its well-known solution is $a=\frac{\left\langle\boldsymbol{r}^{\mathrm{ref}}, \boldsymbol{r}\right\rangle_{L_{2}}}{\left\langle\boldsymbol{r}^{\mathrm{ref}}, \boldsymbol{r}^{\mathrm{ref}}\right\rangle_{L_{2}}}$. Using (5), the corresponding spline projector is specified by $\mathbf{P}^{\text {ref }}=\mathbf{c}^{\text {ref }} \frac{\mathbf{c}^{\text {ref }}{ }^{\mathrm{T}} \Psi}{\left\langle\mathbf{c}^{\text {ref }}, \mathbf{c}^{\text {ref }}\right\rangle_{\Psi}}$, which corresponds to the solution obtained by the direct application of Theorem 1.

\section{ApPliCATION: SEGMENTATION IN BIOIMAGING}

In segmentation algorithms, it is advantageous if prior knowledge about shapes can be integrated [5], [6], [21], for instance in active contour models [1], [11], and [22]-[24]. Our proposed formulation of a projector allows us to compute the distance between a shape and the vector space given by a reference shape. Hence, we can penalize the cases where a shape is distant from the given vector space defined by the reference shape $r^{\text {ref }}$. To illustrate this concept, we consider the example of spline-based snakes [25]. We denote by $E(\Omega)$ the standard energy term that usually needs to be minimized w.r.t. the snake-defining parameters described by $\Omega$. The minimization of $E(\Omega)$ attracts the contour of the snake toward the boundary of the object of interest. We propose to add a prior term to $E$ that we define as $E_{\text {prior }}=\gamma\left\|\boldsymbol{r}-\mathcal{P}^{\text {ref }} \boldsymbol{r}\right\|_{L}^{2}$, where $\gamma \in \mathbb{R}$ controls the contribution of the prior energy term. We re-express $E_{\text {prior }}$ as follows:

$$
\left\|\boldsymbol{r}-\mathcal{P}^{\mathrm{ref}} \boldsymbol{r}\right\|_{L_{2}}^{2}=\left\langle\boldsymbol{r},\left(\mathcal{I}-\mathcal{P}^{\mathrm{ref}}\right) \boldsymbol{r}\right\rangle_{L_{2}}
$$

where $\mathcal{I}$ denotes the identity operator and where we have used the fact that since $\mathcal{P}^{\text {ref }}$ is orthogonal and hence, self-adjoint, then $\left(\mathcal{I}-\mathcal{P}^{\text {ref }}\right)$ is also an orthogonal projector. We use (5) and Theorem 1 to compute the projector. Then, (10) is developed as follows:

$$
\begin{aligned}
\left\|\boldsymbol{r}-\mathcal{P}^{\mathrm{ref}} \boldsymbol{r}\right\|_{L_{2}}^{2} & =\mathbf{c}^{\mathrm{T}} \boldsymbol{\Psi}\left(\mathbf{I}-\mathbf{P}^{\mathrm{ref}}\right) \mathbf{c} \\
& =\mathbf{c}^{\mathrm{T}} \underbrace{\boldsymbol{\Psi}\left(\mathbf{I}-\mathbf{C}\left(\mathbf{C}^{\mathrm{T}} \boldsymbol{\Psi} \mathbf{C}\right)^{-1} \mathbf{C}^{\mathrm{T}} \boldsymbol{\Psi}\right)}_{\mathbf{S}} \mathbf{c}=\mathbf{c}^{\mathrm{T}} \mathbf{S c} .
\end{aligned}
$$

\section{A. Similarity Transformation}

Our solution is generic in the sense that it is applicable to any subclass of the affine family. The case that shows the most
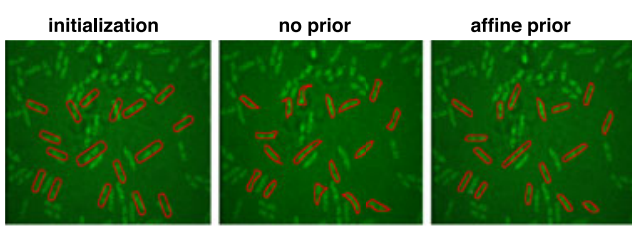

similarity prior initialization

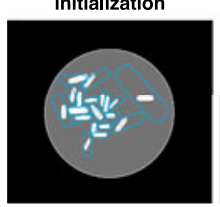

no prior

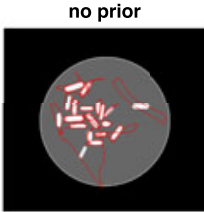

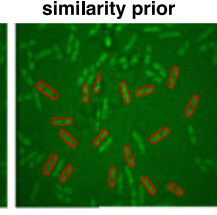

similarity prior
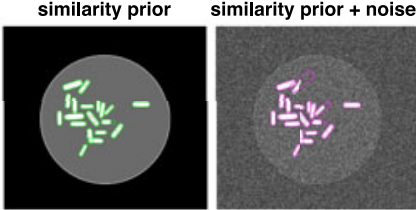

Fig. 1. Segmentation of rod-shaped yeast cells [26] (real data, top; simulated data, bottom). Several snakes are initialized (left) and an image-energy term is optimized with and without prior (affine and similarity). The shape prior corresponds to an approximate rod shape. For the simulated data the gold standard is known and the segmentation results are measured with the Jaccard index (see Table II).

TABLE II

MEAN JACCARD INDICES ON SIMULATED DATA

\begin{tabular}{lcccc}
\hline \hline & Initialization & No prior & Similarity & Similarity + Noise \\
\hline $\mathrm{J}$ & $0.61 \pm 0.17$ & $0.69 \pm 0.25$ & $0.98 \pm 0.4$ & $0.93 \pm 0.14$ \\
\hline \hline
\end{tabular}

potential for biomedical segmentation problems is the new formulation and closed-form solution that we provide for the similarity transformation. In Fig. 1 (top), we illustrate how prior knowledge improves robustness in a segmentation setting, where we compare the affine and similarity transformation. We see that similarity-based shape priors allow for a substantial improvement over the affine transformation of [14]. We also tested the robustness of the similarity prior on simulated data where the gold standard is known (see Fig. 1, bottom) including an image corrupted by Gaussian white noise with $\mathrm{SNR}=0.44 \mathrm{~dB}$. The 19 rod-shaped cells are arranged in clumps, which makes the segmentation more challenging. The computed Jaccard indices (J) are reported in Table II. While our experiments involved quadratic B-splines, we like to point out that our framework is applicable to splines of any order.

\section{CONCLUSION AND SUMMARY}

We provide an explicit closed-form formulation to compute the minimal distance between an arbitrary query curve and a vector space defined by a reference. Our solution is generically characterized for landmark-based spline curves as a projection operator once the basis of the vector space is defined. This allows us to compute the continuous-domain distance as a fast matrix-vector operation. It can be used to efficiently characterize shape priors for landmark-based segmentation models. As application and additional contribution we provide a new direct formulation and implementation of the similarity transform, and we show how it robustifies segmentation algorithms by the integration of prior knowledge. The spline-based solution has the additional advantage that the proposed construction can also be applied to curves that are defined by a set of discrete points or landmarks, by simply interpolating them with a linear B-spline. Our framework can be extended to 3-D tensor-product spline surfaces [27] by noting that the inner product between spline surfaces can also be expressed as a matrix-vector multiplication. 


\section{REFERENCES}

[1] M. Kass, A. Witkin, and D. Terzopoulos, "Snakes: Active contour models," Int. J. Comput. Vis., vol. 1, no. 4, pp. 321-331, Jan. 1987.

[2] V. Caselles, R. Kimmel, and G. Sapiro, "Geodesic active contours," Int. J. Comput. Vis., vol. 22, no. 1, pp. 61-79, 1997.

[3] I. Dryden and K. Mardia, Statistical Shape Analysis. Hoboken, NJ, USA: Wiley, 1998.

[4] B. Li and S. T. Acton, "Active contour external force using vector field convolution for image segmentation," IEEE Trans. Image Process., vol. 16, no. 8, pp. 2096-2106, Aug. 2007.

[5] M. Leventon, W. Grimson, and O. Faugeras, "Statistical shape influence in geodesic active contours," in Proc. IEEE Conf. Comput. Vis. Pattern Recognit., Hilton Head Island, SC, USA, Jun., 2000, pp. 316-323.

[6] M. Gastaud, M. Barlaud, and G. Aubert, "Combining shape prior and statistical features for active contour segmentation," IEEE Trans. Circuits Syst. Video Technol., vol. 14, no. 5, pp. 726-734, May 2004.

[7] Y. Chen et al., "Using prior shapes in geometric active contours in a variational framework," Int. J. Comput. Vis., vol. 50, no. 3, pp. 315-328, Dec. 2002.

[8] T. F. Cootes, C. J. Taylor, D. H. Cooper, and J. Graham, "Active shape models-Their training and application," Comput. Vis. Image Understanding, vol. 61, no. 1, pp. 38-59, Jan. 1995.

[9] T. Cootes, G. Edwards, and C. Taylor, "Active appearance models," IEEE Trans. Pattern Anal. Mach. Intell., vol. 23, no. 6, pp. 681-685, Jun. 2001.

[10] D. Cremers, F. Tischhäuser, J. Weickert, and C. Schnörr, "Diffusion snakes: Introducing statistical shape knowledge into the Mumford-Shah functional," Int. J. Comput. Vis., vol. 50, no. 3, pp. 295-313, Dec. 2002.

[11] A. Foulonneau, P. Charbonnier, and F. Heitz, "Affine-invariant geometric shape priors for region-based active contours," IEEE Trans. Pattern Anal. Mach. Intell., vol. 28, no. 8, pp. 1352-1357, Aug. 2006.

[12] M.-A. Charmi, S. Derrode, and F. Ghorbel, "Fourier-based geometric shape prior for snakes," Pattern Recognit. Lett., vol. 29, no. 7, pp. 897904, 2008. [Online]. Available: http://www.sciencedirect.com/science/ article/pii/S0167865508000238

[13] D. Schmitter and M. Unser, "Similarity-based shape priors for 2D spline snakes," in Proc. 12th IEEE Int. Symp. Biomed. Imag., Nano Macro, Brooklyn, NY, USA, Apr. 2015, pp. 1216-1219.

[14] R. Delgado-Gonzalo, D. Schmitter, V. Uhlmann, and M. Unser, "Efficient shape priors for spline-based snakes," IEEE Trans. Image Process., vol. 24, no. 11 , pp. 3915-3926, Nov. 2015.
[15] D. G. Kendall, "Shape manifolds, procrustean metrics, and complex projective spaces," Bull. London Math. Soc., vol. 16, no. 2, pp. 81-121, 1984.

[16] C. Goodall, "Procrustes methods in the statistical analysis of shape (disc: p321-339)," J. Roy. Stat. Soc. Ser. B, Methodological, vol. 53, pp. 285321, 1991.

[17] E. Klassen, A. Srivastava, W. Mio, and S. H. Joshi, "Analysis of planar shapes using geodesic paths on shape spaces," IEEE Trans. Pattern Anal. Mach. Intell., vol. 26, no. 3, pp. 372-383, Mar. 2004.

[18] A. Srivastava, E. Klassen, S. H. Joshi, and I. H. Jermyn, "Shape analysis of elastic curves in euclidean spaces," IEEE Trans. Pattern Anal. Mach. Intell., vol. 33, no. 7, pp. 1415-1428, Jul. 2011.

[19] D. Bryner, E. Klassen, H. Le, and A. Srivastava, "2D affine and projective shape analysis," IEEE Trans. Pattern Anal. Mach. Intell., vol. 36, no. 5 , pp. 998-1011, May 2014.

[20] M. Unser, "Sampling_50 Years after Shannon," Proc. IEEE, vol. 88 , no. 4, pp. 569-587, Apr. 2000.

[21] G. Charpiat, P. Maurel, J.-P. Pons, R. Keriven, and O. Faugeras, "Generalized gradients: Priors on minimization flows," Int. J. Comput. Vis., vol. 73 , no. 3, pp. 325-344, Jul. 2007.

[22] X. Bresson, S. Esedoglu, P. Vandergheynst, J.-P. Thiran, and S. Osher, "Fast global minimization of the active contour/snake model," J. Math Imag. Vis., vol. 28, no. 2, pp. 151-167, 2007.

[23] A. Blake and M. Isard, Active Contours: The Application of Techniques from Graphics, Vision, Control Theory and Statistics to Visual Tracking of Shapes in Motion, 1st ed. New York, NY, USA: Springer-Verlag, 1998.

[24] F. Precioso and M. Barlaud, "B-spline active contours for fast video segmentation," in Proc. Int. Conf. Image Process., Thessaloniki, Greece, Oct. 2001, pp. 777-780.

[25] R. Delgado-Gonzalo, V. Uhlmann, D. Schmitter, and M. Unser, "Snakes on a plane: A perfect snap for bioimage analysis," IEEE Signal Process. Mag., vol. 32, no. 1, pp. 41-48, Jan. 2015.

[26] D. Schmitter et al., "A 2D/3D image analysis system to track fluorescently labeled structures in rod-shaped cells: Application to measure spindle pole asymmetry during mitosis," Cell Div., vol. 8, no. 6, pp. 1-13, Apr 2013.

[27] D. Schmitter, P. García-Amorena, and M. Unser, "Smooth shapes with spherical topology: Beyond traditional modeling, efficient deformation, and interaction," Comput. Vis. Media, vol. 3, no. 3, pp. 199-215, Sep. 2017. 